\title{
Protée
}

\section{La fiction au péril du récit ? Prolégomènes à une étude de la dialectique entre narrativité et fictionnalité}

\section{René Audet}

Volume 34, numéro 2-3, automne-hiver 2006

Actualités du récit. Pratiques, théories, modèles

URI : https://id.erudit.org/iderudit/014276ar

DOI : https://doi.org/10.7202/014276ar

Aller au sommaire du numéro

\section{Éditeur(s)}

Département des arts et lettres - Université du Québec à Chicoutimi

ISSN

0300-3523 (imprimé)

1708-2307 (numérique)

Découvrir la revue

Citer cet article

Audet, R. (2006). La fiction au péril du récit ? Prolégomènes à une étude de la dialectique entre narrativité et fictionnalité. Protée, 34(2-3), 193-207.

https://doi.org/10.7202/014276ar
Résumé de l'article

Partant du constat d'un emploi difficile du terme " récit " pour décrire les oeuvres contemporaines, cet article examine l'usage de ce terme et en interroge le bien-fondé, notamment dans son rapport avec l'autre paramètre déterminant des textes littéraires, la fictionnalité. Ces deux notions, souvent étudiées conjointement, ne sont pas considérées dans leur dynamique; cette aporie est dépassée grâce à la perspective pragmatique de $\mathrm{R}$. Walsh, qui permet de penser les usages du discours narratif. Depuis un effort de spécialisation des notions de récit (qui correspond au modèle structuraliste canonique) et de narrativité (fondée sur l'événement) est proposée une exploration de certaines modalités de l'instrumentalisation du narratif, une des fonctions qui lui sont communément associées en littérature contemporaine. 


\section{LA FICTION AU PÉRIL DU RÉCIT? PROLÉGOMÈNES À UNE ÉTUDE DE LA DIALECTIQUE ENTRE NARRATIVITÉ ET FICTIONNALITÉ}

RENÉ AudET

Si les expérimentations littéraires des années 1960 et 1970 ont remis en question bien des paramètres associés au discours narratif, la période qui a suivi (et qui constitue, pour l'heure, la période contemporaine) s'est quelque peu réconciliée avec l'objectif de représentation, d'inscription temporelle des actions propre au récit. Cette réconciliation n'est pas pour autant un retour en arrière: les écrivains ne se sont pas résolus à revenir à un usage conventionnel, sage du narratif. Au contraire, on constate, à titre d'indice de cette situation, que toutes les voix critiques célébrant le «retour du récit» ${ }^{1}$ en viennent assez rapidement à moduler cette affirmation teinte d'une fausse nostalgie, laquelle prétendrait à un mouvement inverse, à un retour du balancier vers la zone confortable du récit considéré comme un conventionnel substrat pour le discours littéraire. S’il est de nouveau largement présent dans la littérature actuelle, le récit n'en subit pas moins de spectaculaires expérimentations, de façon similaire à ce qu'on observait dans des œuvres du XXe siècle sinon avant, mais désormais avec une insistance généralisée qui distingue la période contemporaine de toute autre période; le récit est ainsi soumis à des modulations qui le façonnent de manière inusitée ou encore qui le dénaturent - qui le rendent parfois méconnaissable.

Depuis la mise à mal du récit par le Nouveau Roman jusqu'à son épuisement (voir les travaux de Dominique Rabaté [1991]) ou sa raréfaction par une narrativité minimalisée, on se questionne sur l'usage et la fonction du narratif dans les pratiques littéraires des dernières décennies. Ces ouvres ont-elles vraiment cessé de raconter, ou l'ont-elles fait différemment? N'auraient-elles pas plutôt changé de paradigme discursif? Les œuvres semblent bien ne pas se passer du récit, dont la structuration temporelle reste un pilier du travail d'historicisation, de remémoration et de filiation fondamental à la pensée humaine, et constitue une base, dans sa subjectivité, des œuvres contemporaines. C'est davantage du côté de son utilisation qu'il semble falloir s'interroger. On rencontre ainsi constamment des ouvrages fondés sur le discours narratif, mais qui en offrent une lecture, un usage divergent ou étonnant. Depuis un Pierre Yergeau qui découpe sa production romanesque ${ }^{2}$ selon divers épisodes (ou anecdotes), réduisant de la sorte la vectorialisation et la force téléologique du récit, à une Nathalie Sarraute dernière 
manière, qui retourne en fait à son déplacement des actions expérimenté avec ses Tropismes (1939) de début de carrière, qui propose même un détournement des actants (avec des ouvres plus tardives comme Ouvrez [1997], mettant en scène des personnages-mots), la production actuelle offre une gamme de solutions à l'aporie du récit comme réponse universelle au désir d'expression de la psyché humaine. Le plus souvent est investie une zone d'intervention génératrice d'ambiguité, où le texte module la finalité du récit tout en maintenant les repères génériques et discursifs conventionnels - pensons à Gaétan Soucy, dans L'Acquittement (2001), qui décompose la cohérence narrative de son roman en toute fin de parcours ou à Éric Chevillard dont les postures métatextuelles (dans Le Vaillant Petit Tailleur [2003], par exemple) tentent d'embrouiller le lecteur par la multiplication des couches narratives et fictionnelles. Le constat d'une telle variété de façons de construire des œuvres en porte-à-faux sur le récit (entendre: un récit selon les conventions de ce type de discours) nous convainc de la nécessité d'interroger à nouveau la place du discours narratif dans l'économie de l'écriture littéraire actuelle.

Conçu par les structuralistes comme le squelette de la représentation proposée par une œuvre et comme le mode d'articulation des actions qu'elle rassemble, le récit se trouve quelque peu coincé aujourd'hui dans cette acception contraignante, celle-ci le balisant tant dans sa structure intrinsèque (transformation d'états, séquence d'étapes à la Larivaille [1974]) que dans ses moyens de mise en œuvre (durcis par une narratologie que Genette ne souhaitait pas lui-même aussi formalisée). Les dérives que l'on observe dans l'élaboration des récits et les fonctions qui y sont rattachées semblent bien témoigner de cette contrainte théorique tout autant que du poids historique de la pratique narrative. Pour tenter de mieux saisir ces déplacements, nous mettrons ici à l'épreuve une hypothèse qui fait intervenir un paramètre complémentaire, celui de la fictionnalité. Omniprésent dans les pratiques littéraires narratives, le caractère fictionnel est pour ainsi dire télescopé, dans le discours commun, avec la question du récit. Pourtant, les travaux des dernières décennies, tant en théorie narrative qu'en théorie de la fiction, ont vite fait de distinguer - avec raison - la construction d'une œuvre en fonction d'un paradigme temporel et causal et la référentialité des individus logiques qu'elle rassemble. Or, il s'avère que, par un travail de déplacement tout aussi important sur la variable fictionnelle (référentialité floue, frontières du biographique, stratégies métafictionnelles, etc.), les ouvrages contemporains ont rendu cette fictionnalité particulièrement problématique, à l'instar de l'investissement noté pour le discours narratif.

Notre hypothèse se situe ainsi à la conjonction de ces paramètres et de leur dérive: l'utilisation singulière du récit en littérature contemporaine pourrait être le fait d'une fictionnalité problématique des textes, qu'il importerait donc d'étudier conjointement avec le paramètre narratif. En soi, la relation entre fiction et récit apparaît trop peu souvent étudiée en elle-même (les paramètres se confondant dans le discours commun ou se repoussant dans leur saisie théorique); quelques rares propositions en ce sens seront considérées. Cet examen nous conduira rapidement à relativiser la notion de narrativité, à la distinguer de celle de récit, pour lui assigner une extension plus large que celle du terme plus spécifique de récit. C'est donc à une réévaluation de la narrativité que nous procéderons, et de son rapport intime (et mystérieux, il faut le reconnaître) avec la fictionnalité. Le contexte de quelques œuvres contemporaines permettra en fin de parcours d'examiner comment ce rapport peut parfois devenir un rapport de force, imposant à la narrativité un statut instrumental dans certains textes littéraires.

\section{RÉCIT ET FICTION:}

D'UN TÉLESCOPAGE À UNE RELATION INTIME

Parler de fiction narrative, c'est décrire une pratique littéraire majeure, mais c'est surtout faire usage de paramètres, de traits pour saisir un objet. Souvent considérée comme une paraphrase du roman - penserait-on au genre mineur qu'est la nouvelle? -, l'expression «fiction narrative» témoigne bien de l'accointance du fictionnel et du narratif dans les œuvres littéraires. Cette accointance est d'autant plus 
forte qu'elle est forgée à partir d'une relation réciproque de dépendance. La mise en récit appelle fréquemment une mise en situation fictionnelle, qu'expliquerait la trahison inévitable du réel par le langage ou la simple volonté d'inscrire l'anecdote dans l'imaginaire. À l'inverse, l'élaboration d'une fiction prend pour support commun celui de l'intrigue, de l'inscription temporelle et causale du discours narratif, au sein duquel un parcours des contrées fictionnelles peut être proposé. Une telle circularité tend à perpétuer dans le discours la confusion entre les paramètres fictionnel et narratif, d'autant que l'hégémonie romanesque agit comme facteur de maintien de cette perception télescopée.

Depuis quelques décennies, on s'est affairé à identifier et à dénouer ce télescopage. La théorie du récit, s'affirmant avec le structuralisme dès les années 1960, a pris rapidement ses distances avec les pratiques génériques en postulant un fonctionnement autonome du discours narratif; du célèbre Communications 8 (Barthes [dir.], 1966) jusqu'aux propositions d'un JeanMichel Adam (1999), par exemple, la définition du récit s'est élaborée en parallèle des considérations ontologiques ou référentielles. Ces dernières ont en effet été prises en charge par la théorie de la fiction: la sémantique des mondes possibles et les investigations énonciatives sur la nature des actes de langage (depuis John Searle [1969], notamment) se sont occupées de proposer des modèles de définition et d'analyse des incarnations possibles de la fiction dans le langage. Une telle différenciation des paramètres a conduit à une meilleure saisie de leurs traits et manifestations, sans compter une compréhension approfondie de leurs enjeux respectifs. Si la conceptualisation des notions impliquées est parvenue à opérer une scission nette dans le métadiscours littéraire et philosophique, les objets demeurent néanmoins l'incarnation perpétuée de cette collusion entre récit et fiction. La réconciliation entre les approches théoriques et les objets culturels et littéraires - étape inévitable, voire obligée - survient de façon plus marquée dans la mouvance des travaux historiographiques de Hayden White (1975), de sa réflexion sur la narrativisation de l'histoire. À sa suite, Paul Ricœur (1983) jette des ponts entre la littérature, l'histoire et des propositions fondamentales sur la temporalité du discours narratif; de même Gérard Genette interroge-t-il la frontière entre récit factuel et récit fictionnel (1991), dans une approche qui rejoindra celle de Dorrit Cohn dans The Distinction of Fiction (1999) et dans la continuité de Logique des genres littéraires (1957) de Käte Hamburger.

Ces modalités de dialogue entre récit et fiction confortent une fois de plus le recours au tableau à double entrée, voyant dans ces paramètres deux traits à associer selon une logique combinatoire. On a ainsi longuement discuté de la fictionnalité ou de la nonfictionnalité de textes empruntant la forme de récits, comme les travaux de Genette, Adam et Cohn l'illustrent bien; on a certainement pris acte de la case où se rencontrent non-récit et non-fictionnalité, sans pour autant qu'elle soit investie de façon notable par les théoriciens du récit (cette prose blanche appartenant davantage au discours argumentatif, au mieux à la description). S'il fallait faire le procès de ce tableau, on notera l'absence à peu près complète de discussions sur une autre case, celle par laquelle on soutiendrait qu'une fiction pourrait exister en dehors du récit. Il jaillit clairement de ce portrait épistémologique deux observations principales. La première tient au fait que la réflexion sur la dialectique propre de la fiction et du récit tourne court - elle se résume généralement à la définition des marques fictionnelles au sein d'un discours narratif (l'ouvrage de Cohn est emblématique de ce point de vue). En fait, il ne s'agit pas tant de l'étude d'une dialectique que de l'identification du mode d'inscription de la fictionnalité dans le discours (narratif, en l'occurrence). Se confirme ainsi, de facto, l'inefficacité du recours au tableau à double entrée: ces deux paramètres décrivent des réalités ou des traits qui n'ont pour base commune que l'objet dans lequel ils s'incarnent. Il ne faut pas voir là une aporie purement théorique, contredite par les objets culturels mêmes qui illustrent à l'évidence la possibilité d'une cohabitation du récit et de la fiction. Le problème réside dans le choix de l'angle d'étude de leur dialectique; nous y reviendrons. La seconde observation suscitée par ce portrait des travaux sur le 
rapport entre le discours narratif et la fictionnalité porte sur la prédominance de celui-là sur celle-ci: dans le regard critique que l'on jette sur les ouvrages de fiction narrative, sur leur dynamique interne, il existe toujours un monopole de la perspective narrative, contexte à partir duquel on cherche les incarnations du fictionnel. Ce regard est orienté notamment du fait qu'il constitue le principal (parce qu'unique) champ d'investigation de la dialectique récit-fiction: si les théories du discours narratif se sont engagées sur le terrain de la fiction narrative, les théories de la fiction (moins poétiques et plus philosophiques) gardent une réserve et se restreignent à leur objet d'étude - d'où cette absence de la perspective fictionnaliste sur le récit ${ }^{3}$. De ces deux observations émerge donc le constat de la nécessité d'interroger conjointement le récit et la fiction dans leurs rapports afin d'en arriver à une meilleure compréhension du fonctionnement des textes littéraires.

En dehors du discours canonique sur le récit ont été avancées des propositions qu'on qualifiera de stimulantes, à tout le moins pour le coup de barre qu'elles imposent à l'orientation des travaux sur les paramètres qui nous intéressent ici. La première perspective à convoquer est celle, aussi étonnante soitelle, de la sémantique des mondes possibles. Puisant dans le substrat philosophique de la logique des mondes possibles développée par Leibniz, elle se construit en formulant diverses conditions régissant l'existence des mondes, en questionnant les différentes modalités intervenant dans leur élaboration. Dans une évaluation a priori de cette approche, on pourrait être tenté d'y voir une modélisation autonome de la fiction. C'est notamment ce que nous laisse croire la typologie des mondes possibles établie par Lubomír Doležel, dont le premier type, le "world of states" - dit W(S) -, se caractérise par l'absence d'action, d'actant et donc de récit: «W(S) est un monde parménidien, clos et atemporel, un monde d'immobilité et de silence où rien ne change, où rien ne se produit» ${ }^{4}$. Toutefois, l'impression s'évanouit aussitôt, car les trois autres types se caractérisent par l'inclusion de diverses entités et structures qui ont pour effet de faire de ces mondes des narrative worlds. Doležel considère en effet, contrairement au discours des théoriciens de la lignée de Hamburger, Genette et Cohn, que c'est par la structuration et l'ameublement d'un monde que l'on crée un récit, les mondes fictionnels étant à lire comme l'équivalent de macrostructures narratives. De façon plus spécifique, il envisage les mondes fictionnels des récits dans leur constitution, dans leur genèse, dont la première étape est la sélection (la détermination des entités et le choix de la catégorie à laquelle appartient le monde) et dont la seconde, "opération de configuration, modèle les "narrative worlds" selon un ordre qui permet de produire, de générer des "stories" ". Nous sommes donc ici dans une perspective diamétralement opposée à celle de Genette et consorts: si ceux-ci considéraient d'emblée le texte narratif et y recherchaient des marques de fictionnalité qui pourraient le faire basculer d'un régime référentiel à un régime fictionnel, Doležel et autres partisans de la sémantique des mondes possibles envisagent plutôt le texte dans le processus qui le voit naître, où c'est la volonté de mettre en place un monde (à la limite qu'il soit réel ou imaginaire) qui conduit, dans son organisation interne, à la production d'une trame narrative.

Cette conception théorique intégrée du récit et de la fiction est encore plus nette dans les travaux de théoriciens comme Marie-Laure Ryan (1991) et David Herman (2002), dont l'allégeance à la sémantique des mondes possibles est notoire, même si les travaux cités s'inscrivent explicitement en théorie du récit. Participant de la théorie de la fiction et du discours narratif, leurs réflexions se structurent autour d'une notion clé, non celle de fictional ou narrative world, mais bien celle de storyworld. Cette notion, qui se définit de façon large comme «la classe de modèles discursifs qui permet de comprendre un discours organisé narrativement ${ }^{6}$, déplace le principe de la logique des mondes possibles dans le cadre interprétatif rattaché au discours narratif: la compréhension de ce que met en scène un récit passe donc par des alternative possible worlds, lorsque le monde représenté pouvait être fictionnel ou être un monde possible qui correspond au monde actuel mais dans un état antérieur. Dépassant la stricte question 
des relations entre mondes, cette transposition dans le contexte des textes narratifs permet à la notion de storyworld de renvoyer à l'«écologie de l'interprétation narrative" (ecology of narrative interpretation):

En voulant donner un sens à un récit, les lecteurs tentent de reconstruire non pas uniquement ce qui a pu arriver, mais aussi le contexte ou l'environnement encadrant les individus $d u$ "storyworld», leurs attributs et les actions et événements auxquels ils ont participé. 7

Une telle capture du monde fictionnel de l'intérieur du récit inverse en quelque sorte la dynamique en place chez Doležel, car il s'agit, chez Ryan et Herman, d'un retour en force du cadre perceptuel narratif. La grammaire narrative absorbe ici la sémantique des mondes possibles pour expliquer, signale Herman, l'immersion du lecteur dans le monde représenté par le récit, pour expliquer le sentiment de partager un univers avec les personnages qui le meublent. Par cette perspective cognitiviste, nous sommes revenus en fait au point de départ du monopole narratif sur la fiction - à une nuance près, celle d'une approche totalement différente de la question de la fictionnalité des référents.

Si l'on quitte le champ cognitif et poétique qui jusqu'à présent caractérise les travaux cités, on peut trouver une proposition très intéressante du côté des articles de Richard Walsh, dont la lecture de la fictionnalité et du discours narratif transite par des préoccupations complémentaires. Revisitant dans un premier temps la notion fondamentale de la fabula, Walsh montre bien à quel point, pour les Formalistes russes, la fictionnalité et la fabula étaient intimement intriquées, et de quelle façon ce lien par trop évident a été déclassé par les narratologues structuralistes:

Les Formalistes considéraient que le récit fictionnel était principalement défini par les règles de l'art (peu importe la façon dont on pouvait les concevoir), et que son adhésion à une logique représentationnelle était toujours, d'une certaine façon, masquée. Les conséquences de cet intérêt spécifique pour la fictionnalité ont été oubliées dans les développements ultérieurs de la théorie $d u$ récit, précisément en raison du fait que la littérarité a été perçue comme un attribut du récit en soi. 8
Walsh relève ainsi un double déplacement: le glissement pernicieux de caractéristiques de la fiction narrative vers le récit en lui-même, phénomène de généralisation abusive, de même que l'effacement de considérations sur la fictionnalité des textes (sur leur portée représentationnelle) au profit d'une systématisation à l'envi des procédés narratifs. Poursuivant ailleurs sa réflexion en montrant comment la fabula (qui correspond à peu de choses près à la notion d'histoire chez Genette) constitue non une donnée factuelle, une histoire préexistant au récit, mais bien une inférence du lecteur (un «by-product of the interpretative process»), il en vient peu à peu à détacher fictionnalité et récit, rappelant le caractère artificiel du récit: «les récits sont des constructions [sémiotiques], et leurs sens sont définis de l'intérieur du système narratif" 9 .

Par cette orientation de son propos vers les enjeux communicationnels de la prose narrative, Walsh délaisse les considérations poétiques ou ontologiques de la fiction au profit d'une lecture pragmatique. La fiction, placée dans une logique communicationnelle, apparaît selon lui comme un attribut du langage: un attribut fonctionnel (il s'agit d'un certain usage du langage) et un attribut rhétorique (dans l'appel adressé à l'«interpretive attention" du lecteur).

La fictionnalité, propose-t-il, fonctionne comme un cadre communicationnel: elle repose sur un usage du langage, et son caractère distinct réside dans une structure rhétorique spécifique, reconnaissable comme telle, qui est appelée par cet usage. 10

Perçue ainsi, la fictionnalité se construit d'abord parallèlement au récit: ils sont tous deux des paramètres de la mise en forme d'un message en fonction de la production d'un certain effet - générer une prose narrative en régime fictionnel. Ce caractère très pragmatique de la communication littéraire ne se restreint pas au simple constat de la cohabitation circonstancielle de la fiction et du récit; Walsh poursuit en effet en montrant que l'élaboration de la prose narrative est fonction de sa lecture et de son caractère processuel. Partant des propositions de Mary Louise Pratt (1977) qui revisite la théorie des actes de langage pour l'appliquer au discours littéraire, il s'engage dans une perspective résolument 
pragmatique. Si l'on considère le texte littéraire sous l'angle des règles conversationnelles et du principe de coopération de H.P. Grice (1975), les maximes fondamentales du discours littéraire sont celles de la pertinence et de la qualité. Cette qualité ( ne dites pas ce que vous savez être faux" ["do not say what you believe to be false"]), qui pour résumer grossièrement correspond pour Walsh au paramètre fictionnel, devrait être perçue comme étant subordonnée à la pertinence ("be relevant"), moteur réel du texte (2005: 155-158).

Comment comprendre alors ce principe de pertinence dans un texte littéraire? Mary Louise Pratt l'avait associé à une caractéristique pragmatique du texte: sa racontabilité (to be tellable), son intérêt à être raconté. Or, rappelle Walsh en convoquant Sperber et Wilson (1984), la traversée d'un discours se fonde sur un processus inférentiel, la lecture projetant une série d'hypothèses au fil de son avancée; ainsi en est-il de l'évaluation de la "qualité» d'un texte, à savoir son caractère fictionnel, qui se saisit grâce à des inférences portant sur la référentialité du discours (le personnage évoqué par les indices qui me sont donnés jusqu'à ce point de ma lecture renvoie-t-il à un individu du monde réel ou appartient-il à un monde imaginaire?). Toutefois, ce processus inférentiel suit la cadence du texte, et s'y conforme:

$$
\begin{aligned}
& \text { La pertinence de la fiction n'est pas validée globalement, de façon } \\
& \text { analogique, mais plutôt de façon incrémentielle, composant avec } \\
& \text { l'apport de diverses données cognitives qui ne sont mobilisées qu'au } \\
& \text { moment de l'acceptation de la véracité de la proposition elle-même, et } \\
& \text { qui ne le sont que par le déploiement, l'investissement et l'intégration } \\
& \text { de ces données dans la forme narrative. } 11
\end{aligned}
$$

C'est dans ce caractère incrémentiel que Walsh situe l'interaction fine entre la fiction et le récit, dans une dynamique de la résolution repoussée - résolution non pas narrative, mais bien pragmatique.

Il y a certainement, poursuit-il, une perception globale et rétrospective qui permet de saisir le récit comme la mise en sursis, tout au long du fil narratif, du jugement de sa pertinence; dans ce contexte, la clôture narrative apparaît moins comme la résolution de l'intrigue (bien que ce soit le résultat habituel du point de vue de l'intrigue) que comme la résolution des évaluations de pertinence jusque-là suspendues. Selon cette perception, détachée des enjeux fictionnels, la forme narrative répond en elle-même à des attentes de pertinence. 12

D'emblée, le discours narratif peut sembler mis au service de la fiction (en servant de support à la construction feuilletée et progressive de l'univers fictionnel); mais, par cette perspective rétrospective, on en vient rapidement à concevoir conjointement leur élaboration à travers le jeu de tensions mis en place par la combinaison des maximes de qualité et de pertinence de la communication littéraire. Si la conception initiale de la fiction proposée par Walsh nous enjoignait, dans un premier temps, d'envisager minimalement une construction parallèle de la fictionnalité et du récit, force nous est d'admettre avec lui que cette construction est bien davantage conjointe, voire réciproque: alors que la fiction se bâtit grâce au caractère progressif du récit qui tend vers sa résolution, le discours narratif s'élabore en fonction des vides fictionnels qui assurent une racontabilité au texte (par le fait de repousser en fin de parcours l'évaluation de la fictionnalité des référents). Une telle proposition d'analyse du discours littéraire, fondée sur ses enjeux pragmatiques, offre un regard étonnamment neuf sur les conditions de mise en place de paramètres comme la narrativité et la fictionnalité, tout en écartant le monopole d'un facteur au détriment de l'autre.

\section{RÉCIT OU NARRATIVITÉ?}

Les propositions de Richard Walsh semblent conduire à une compréhension nouvelle de la fictionnalité et de son rapport avec la prose narrative: envisagés dans leur dimension communicationnelle, ces paramètres se révèlent dans leur fonction d'attributs du discours littéraire. Ils se trouvent ainsi dégagés - à tout le moins du point de vue théorique des connotations rattachées aux pratiques génériques et des tentatives de saisie qui ne se basent que sur les manifestations discursives de ces paramètres. Plus encore, il paraît ici important de signaler un apport indirect de ces propositions, qui réside dans un implicite que Walsh ne développe pas (et qu'il n'endosserait peut-être pas d'ailleurs, car telle n'est pas 
sa visée). Cet implicite concerne plus particulièrement la conception du récit sous-tendue par cette interaction fiction-récit dans une perspective communicationnelle. Walsh montre bien que l'évaluation de la pertinence de la fiction, de sa transmissibilité (pour éviter le terme connoté de «racontabilité»), repose sur la projection inférentielle à travers le texte. Cette projection est le résultat d'un ensemble d'inconnues qui parsèment ce texte, lequel agit comme instance de la «mise en sursis, tout au long du fil narratif». C'est là,

rétrospectivement, la fonction associée au récit: agir comme retardateur, comme trame supportant ces vides, ces désirs de saisie entière de la pertinence d'une fiction. L'intrication de nos deux paramètres est donc fondée sur une exigence, celle de cette "line of action». Toutefois, la résolution de l'intrigue n'apparaît pas comme un critère déterminant (elle peut ou non survenir); c'est la clôture du texte qui importe davantage, cette terminaison appelant la fin des inférences et la levée de la suspension de l'évaluation de pertinence du texte (le caractère transmissible de sa fictionnalité). Il est donc possible qu'il y ait coïncidence entre la résolution de l'intrigue et l'établissement, en fin de parcours, de la pertinence du texte; elle n'est cependant pas significative dans l'interaction entre fiction et récit.

L'exigence de la combinaison fiction-récit à l'endroit de la trame narrative ne vise donc pas sa dimension téléologique, sa réalisation selon le modèle idéalisé du récit (la schématisation typique proposée par Bremond ou Larivaille, par exemple), où la tension mise en place en ouverture trouve sa résolution après une complication et une série d'actions; cette exigence est déplacée selon une visée plus fonctionnelle du texte: une exigence communicationnelle. Il n'y a donc pas, dans ce modèle, obligation pour le récit de se conformer aux principes d'une grammaire narrative la fiction peut s'inscrire dans un texte s'y conformant (c'est là le cas de figure le plus commun), mais ne nécessite pas cet ancrage dans une vectorialité événementielle qui pousse le récit vers sa fin. La perspective pragmatique de Walsh appelle plutôt une dynamique narrative: celle-ci, qui ne se fonde pas sur le canon de la structuration des actions dans un récit, repose sur le seul engagement dans le texte d'un mouvement d'ordre narratif.

Quel impact associer à cette nuance qui peut sembler bien ténue? La lecture de Richard Walsh révèle bien que la fiction ne nécessite pas la mise en place d'un récit comme tel, d'un récit dans sa conception forte et canonique. La fiction appelle seulement un support combinant représentation d'actions et temporalité, ce support permettant de reporter la résolution des inférences laissées en plan jusqu'au moment où il ne sera plus possible de les repousser - au moment de la clôture matérielle du texte. Il faut en déduire que la fictionnalité exige sa coconstruction non pas avec un récit, mais avec certaines caractéristiques de ce récit, parmi lesquelles ne figure pas l'obligation d'une téléologie, d'une structuration forte et vectorialisée en fonction d'une résolution narrative. Sur la base de cet implicite de la proposition de Walsh et à partir des observations (citées en introduction) sur l'évanescence du récit dans les productions contemporaines, il apparaît nécessaire d'en arriver à un questionnement du référent de la notion de récit et de son équivalence problématique avec le terme voisin, mais combien vaporeux, de narrativité.

Si l'on cherche d'emblée à saisir la portée du terme de narrativité, on découvre assez rapidement son inadéquation à parler clairement d'une réalité. Son acception relève manifestement plus du discours commun que de la terminologie scientifique, plus stricte dans son référent. Renvoyant généralement à l'idée du récit, la narrativité ne semble décrire que l'existence désincarnée des textes narratifs dans l'ensemble qu'ils forment (la narrativité dans tel corpus) ou encore la présence d'un récit dans un texte donné (la narrativité de telle ouvre), l'une et l'autre notions se révélant donc plus ou moins des synonymes. L'utilité du terme paraît ainsi se restreindre à une voie de contournement linguistique pour éviter la redite.

Du côté des théoriciens du récit, dans leurs écrits et dans les ouvrages de référence, la narrativité semble encore moins prisée, tant elle échappe communément aux recensions terminologiques. Elle surgit néanmoins 
à quelques reprises; nous en évoquons ici trois manifestations. Greimas et Courtés, dans leur Dictionnaire raisonné de la théorie du langage, proposent pour le terme une entrée témoignant bien des présupposés qui soutiennent leur conception de la sémiotique. Leur définition, en fait, présente d'abord la narrativité comme un critère discriminant, qui permet la distinction entre les discours narratifs et les discours non narratifs; ils en viennent néanmoins assez rapidement à la nomenclature des caractéristiques propres au discours narratif (du point de vue de l'énonciation, de la structuration des textes). $\mathrm{Si}$, de cette façon, Greimas et Courtés préparent le terrain pour des propositions de définition voisines (comme on le verra plus loin), ils aboutissent à une conception ouverte de la narrativité:

Dans le projet sémiotique, qui est le nôtre, la narrativité généralisée - libérée de son sens restrictif qui la liait aux formes figuratives des récits - est considérée comme le principe organisateur de tout discours. (1979 : 249)

Ainsi présentée, la notion pourrait presque être un gage de littérarité, voire constituer le paramètre fondamental de la littérarité - à tout le moins est-ce là, pour eux, la base de la discursivité, qualité de ce qui fait d'un texte un discours. C'est le signe le plus tangible de l'ambition de la sémiotique greimassienne, dont les principes permettraient de saisir dans son entièreté la diversité des pratiques sémiotiques.

Consacrant un ouvrage entier à la théorie et à la mise en œuvre de la narrativité, Philip J.M. Sturgess n'en demeure pas moins fidèle aux orientations de Greimas et, d'une certaine façon, de Gerald Prince (qui constituera le troisième coup de sonde, un peu plus bas). Sa conception, faut-il le souligner d'emblée, n'apparaît pas révolutionnaire. Passant en revue diverses propositions théoriques sur le récit (RimmonKenan [1983], Scholes [1982], Prince [1973] ...), il tente de prendre position en évitant les écueils rencontrés par ses prédécesseurs et les vides laissés par les avancées théoriques antérieures. Le résultat est d'abord celui d'un énoncé de principe: la narrativité est la capacité d'une œuvre à être narrative. L'idée de la narrativité comme qualité du texte rejoint assurément la proposition de Greimas et Courtés; Sturgess toutefois se démarque par sa façon d'évaluer cette qualité. Il considère en effet les textes sous l'angle de ce qu'on pourrait appeler une économie narrative - la gestion interne de la configuration du récit:

La narrativité renvoie principalement à la façon dont s'articule un récit, à la façon dont chaque partie de développement engendre une crise ou un dilemme dans le discours qui se résout par sa propre continuation, peu importe la forme que celleci emprunte. 13

Les modalités de l'articulation interne du récit correspondent donc au mouvement de la narrativité, selon Sturgess, qui ouvre ainsi la porte à différents types de narrativité, à différentes expressions de la narrativité; conçue comme "la force agissante du récit, une force qui opère constamment dans le récit, de façon syntagmatique» ${ }^{14}$, elle ferait émerger la singularité d'un récit dans son arrangement et dans sa réalisation. Un tel accent sur la spécificité des textes constitue une posture pour le moins difficile à soutenir, puisqu'il n'existe guère de généralisation possible (sinon le fait que tous les textes considérés exploitent le discours narratif). Il en vient même à rejoindre Greimas et Courtés en voyant, dans cette notion, l'accès possible à une lecture du geste narratif en fonction du sens à donner au texte:

$$
\begin{aligned}
& \text { [...] lire un récit en prenant sa totalité en considération } \\
& \text { correspond au fait de lire sa narrativité. Cette lecture engendre } \\
& \text { elle-même une histoire - l'histoire de la narrativité - qui vient } \\
& \text { augmenter l'histoire, renforcer sa force dramatique et par-dessus } \\
& \text { tout apporter un sens à l'histoire racontée. } 15
\end{aligned}
$$

Parallèlement à une telle proposition ancrée dans l'absolu sémantique du texte, Prince tente une approche immanente du texte, fondée sur des caractéristiques plus aisément reconnaissables. Dans son Dictionary of Narratology (qui sert de base à sa réflexion, reprise par la suite dans l'entrée qu'il signe dans The Routledge Encyclopedia of Narrative Theory), il avance une conception double de la narrativité: "L'ensemble des caractéristiques définissant le narratif et le distinguant du non-narratif; les traits formels et contextuels qui rendent un texte (narratif) plus ou 
moins narratif» ${ }^{16}$. Il y a donc juxtaposition de deux acceptions: la qualité du texte (à la façon de Sturgess et de Greimas) et les conditions d'élaboration de cette narrativité, un texte pouvant être évalué en fonction de son degré de narrativité. Prince convient néanmoins que ces deux volets se recoupent et éludent certaines questions, mais s'en tient tout de même à l'idée fondamentale d'une gestion interne des procédés:

La narrativité semble liée fondamentalement à la question de la configuration narrative d'ensemble (par opposition à des traits thématiques spécifiques, à une visée contextuelle ou à une valeur textuelle). Plus précisément, elle semble liée à la dimension formelle du narré (par exemple la clôture structurelle) plutôt qu'à celle du narrant (par exemple la mise en évidence de la singularité des événements) et aux relations existant entre les deux (par exemple la présence de commentaire en proportions raisonnables). 17

La narrativité en vient ainsi à couvrir toutes les modalités d'application du discours narratif dans le texte, de l'évaluation de son importance relative jusqu'à la prise en compte des procédés mobilisés.

Au terme de cette exploration sommaire, quel sens pouvons-nous associer à la notion de narrativité? Les exemples cités nous conduisent presque à la considérer comme la science du récit, comme perspective sur les conditions d'incarnation du récit dans un texte sinon, nous en venons à déduire que la narrativité, telle que nous l'enseigne le discours commun, redouble la notion de récit. Y a-t-il réel chevauchement des deux termes? Sans refaire ici l'exercice d'une analyse des propositions de définition du terme de récit, on peut néanmoins mettre en lumière les constantes permettant d'en baliser la conception canonique. L'intégration, dans une séquence configurée (pour reprendre le terme de Ricœur) qu'on nommera intrigue, d'actions qui participeront d'un effet de téléologie permet la création d'un récit, en supposant leur inscription dans une temporalité fondamentale, l'assignation des actions (la présence d'un sujet agissant) et le recours à une voix qui puisse transmettre de l'intérieur une perception de ces actions. La traversée des ouvrages théoriques sur le récit révèle assez rapidement l'obsession pour les actions et leur configuration, leur enchaînement trace ici des réflexions pionnières des structuralistes qui continuent d'orienter fortement les études sur le discours narratif. D'autres avenues sont certes exploitées; pensons aux travaux sur la voix narrative qui occupent les analystes du roman contemporain, de même qu'à la somme théorique d'un Ricœur consacrée à la temporalité. Néanmoins, le récit demeure captif d'une définition extrêmement rigide, étant perçu dans sa manifestation idéale - ce que reconnaissent, en fin de parcours, les théoriciens, qui ne manquent pas de signaler la nécessité d'adaptation des modèles proposés à l'incarnation bien singulière du récit dans les œuvres littéraires.

Pour tenter de contourner cette aporie et d'ouvrir la réflexion à une autre perspective sur le discours narratif, nous suggérons ici, à la lumière de l'implicite présent dans les réflexions de Richard Walsh, le décentrement du mode d'approche du récit. Dans la continuité d'un article consacré à cette question (Audet, 2006), nous proposons de distinguer le récit de la narrativité, de spécialiser ces termes afin d'en arriver à une compréhension plus ouverte des phénomènes narratifs. Si l'on maintient le récit non dans son acception commune mais comme terme désignant la structure narrative organisée et incarnée (basée sur les schémas à épisodes des structuralistes), il serait intéressant de proposer une conception plus large, moins téléologique, de la narrativité. Elle se détacherait ainsi des enjeux configurationnels propres au récit pour désigner plus globalement ce mouvement d'ordre narratif qui émergeait des réflexions de Richard Walsh. Nous avons ainsi proposé de définir la narrativité non comme une forme minimale (minimaliste) du récit, mais en fonction d'une perspective plus phénoménologique: la narrativité se fonde sur l'événement ${ }^{18}$. Recourir à la notion d'événement n'est pas une ruse pour simplement renommer les events et les happenings, tels que les actions sont classifiées par les chercheurs anglo-saxons; c'est plutôt l'occasion de reconnaître dans l'événement une potentialité narrative du fait de sa fulgurance, de son surgissement dans un contexte où il n'était pas 
attendu. Pour qu'il y ait narrativité, il ne s'agit pas de dénombrer des unités (des actions, des événements ${ }^{19}$ ); il faut donc, dans cette perspective, qu'il y ait événement.

La distinction que nous proposons d'établir entre récit et narrativité a pour avantage de décentrer la réflexion sur le narratif de sa stricte incarnation textuelle - à laquelle est ici dédiée la notion de récit. Par ailleurs, cette conception de la narrativité basée sur son événementialité permet de rendre compte de phénomènes littéraires où le récit n'est pas réalisé en regard de son modèle idéal, donc de ne pas être constamment obligé de décrire le discours en termes dépréciatifs. Le recours fréquent à des formules comme le minimalisme narratif (qui existe certes, mais de façon plus spécifique que l'usage fait de cette expression) ou le récit en crise témoigne de l'efficacité limitée des termes communément employés. Il y a donc place pour une saisie des manifestations narratives en dehors de l'évaluation de leur conformité avec le modèle canonique, ce à quoi peut contribuer la narrativité dans la définition qui est ici proposée.

Cette conception de la narrativité permet également d'envisager plus finement la dialectique que le mode narratif entretient avec la fictionnalité. Cette finesse repose sur une capacité à entrevoir les variations possibles dans la relation entre ces deux paramètres - c'est en effet la géométrie variable de cette dialectique qui ouvre à une diversité des représentations proposées dans les œuvres littéraires. Si le rapport narratiffiction peut être fortement influencé par la manière dont s'élabore un monde fictionnel, il dépend tout autant, sinon davantage, des usages de la narrativité. Dans sa dialectique avec le fictionnel, la narrativité peut jouer différents rôles, peut se voir attribuer diverses fonctions (dont l'inventaire reste manifestement à dresser). En guise de clôture à cette réflexion exploratoire, un cas de figure sera évoqué: l'instrumentalisation du narratif.

\section{LA NARRATIVITÉ INSTRUMENTALISÉE}

Si la pratique romanesque nous a habitués à une cohabitation équitable du fictionnel et du narratif (l'un complétant l'autre, selon le modèle proposé par
Walsh), nombre d'œuvres nous conduisent à réviser nos attentes et à associer un rôle différent au discours narratif. Certaines pratiques fictionnelles déclassent le narratif - mais ce déclassement ne doit pas être vu comme une réalisation imparfaite ou minimale du récit. Lorsque Jean-Michel Adam travaille à distinguer les gestes de raconter et de relater, il ne fait pas état d'un usage différent du narratif (à tout le moins n'estce pas là son objectif); présentant deux types de représentation des actions, le premier étant structuré par une intrigue et non le second, il vise en réalité à définir les conditions minimales d'existence du récit. Ainsi la description d'actions ( relater») ne rencontret-elle pas les «critères de définition d'une forme de mise en texte et de représentation des actions qu['il] propose d'appeler "récit" (1994: 20). Lisant les manifestations textuelles de la narrativité en fonction de son incarnation idéale sous la forme d'un récit, Adam ne considère pas la fonctionnalité de cette description d'actions, car sa perspective linguisticodiscursive a une visée normative. Le déclassement du narratif par rapport à la fictionnalité d'un texte renvoie plutôt à des pratiques qui déjouent la fonction structurante du récit, reléguant ainsi la dimension narrative à un rôle instrumental. Agissant comme un support de la fiction, inévitablement, la narrativité n'organise pas le discours, le sens de celui-ci n'étant pas associé à un développement de type causal et temporel - la signification d'un texte ne se construisant pas en fonction de la téléologie du récit, de son éventuelle résolution narrative.

Quelques pratiques littéraires se spécialisent dans le bouleversement des attentes liées à la dialectique entre fiction et narrativité ${ }^{20}$. Avec l'essai, par exemple, nous sommes en présence d'une pratique qui ne se situe pas d'emblée en régime fictionnel, pas plus qu'elle ne relève pleinement d'un discours de type narratif. Toutefois, il n'est pas rare que certains textes dans un recueil d'essais, que certains passages à l'intérieur d'un essai fassent la part belle à la fiction ${ }^{21}$ : l'affabulation, aussi problématique soit-elle dans ce contexte, aura alors fonction d'exemplum ou soutiendra le parcours réflexif en mettant en scène une contrepartie de l'essayiste (pensons à J. Brault dans Ô saisons, ô 
châteaux [1991]). Si la fiction conduit à inscrire des actions dans une séquence temporelle, l'achèvement de cette séquence narrative n'apparaît pas signifiante en elle-même: la démonstration (si une leçon explicite peut en être déduite) récupère plutôt la situation présentée au profit d'une hypothèse de lecture d'un fait culturel, par exemple. Le discours narratif opère donc comme support de la fiction, mais sans nécessairement qu'il se réalise sous la forme d'une intrigue, ni que cette éventuelle configuration événementielle porte quelque valeur de racontabilité, pour reprendre le terme élaboré par les pragmaticiens.

Il est possible d'observer un tel déplacement du rapport entre fiction et narrativité dans certaines pratiques de la littérature électronique, notamment les hypertextes de fiction. Ces œuvres, en se calquant jusqu'à un certain point sur la prose narrative conventionnelle (dans son objectif de représentation d'actions en régime fictionnel), suscitent donc des attentes qui sont conformes à la pratique romanesque: structuration temporelle, configuration des actions, non-référentialité des individus logiques convoqués, etc. Pourtant, c'est justement cette supposée équivalence qu'investissent les hyperfictions. Poursuivant l'effort de projection dans un monde alternatif, elles en arrivent toutefois à déconstruire profondément toute velléité d'architecture narrative un tant soit peu achevée. En jouant de la rupture (textuelle, temporelle et donc narrative), ces œuvres électroniques proposent une lecture différente de la fiction, qui ne se fait pas à l'aune d'une éventuelle résolution narrative - qui, si elle advenait, ne pourrait coïncider avec une terminaison textuelle, absente par le fait même de la configuration réticulaire d'un hypertexte. S'il y a, à l'œuvre, dans le geste d'écriture des auteurs de littérature électronique, une déconstruction volontaire du récit, le résultat, lui, laisse au lecteur une narrativité éclatée, fondée sur une série de surgissements disséminés, à la limite sur un non-événement (ou un événement qui refuse d'advenir), d'où la fréquente allusion aux errances et désillusions des lecteurs devant ces œuvres déboussolantes ${ }^{22}$. La narrativité des hyperfictions, bousculée en comparaison avec les romans-étalons dans la lignée desquels ils se situent, revêt une fonction tout autre, rhétorique: le monde que vous, lecteurs, tentez de saisir ne peut s'inscrire dans la traditionnelle logique fondée sur la causalité et la temporalité.

Dans ces deux pratiques, la fonction associée à la narrativité se trouve génériquement déterminée: soit le discours narratif n'appartient pas au personnel fondamental du genre, comme dans le cas de l'essai, soit il ne peut se réaliser sous sa forme canonique du récit pour des raisons formelles et esthétiques, l'hyperfiction illustrant bien ce cas de figure. On se retrouve ici en présence de deux manifestations d'une narrativité instrumentalisée, la fiction prenant le haut du pavé en devenant le principal support du sens. $\mathrm{Si}$ ces textes demeurent néanmoins narratifs, cette inscription temporelle et éventuellement causale d'actions ne pourra vraisemblablement servir qu'à rendre possible l'élaboration d'une fiction.

La négociation qui s'opère entre la fictionnalité et la narrativité peut également se manifester par des jeux de tension plus subtils où la manipulation des horizons d'attente joue un rôle déterminant. Prenons pour exemple le cas de certaines pratiques polytextuelles. Le rassemblement de textes brefs en un recueil n'est pas un geste sans conséquence; les études sur la poésie, l'essai et la nouvelle l'ont illustré à de nombreuses reprises et sous des angles extrêmement divers. Dans les études sur les recueils de nouvelles, on a fréquemment noté que la mise en recueil permet de mettre en valeur une dimension des textes que leur lecture isolée ne révélait que difficilement: thématique dominante, constantes dans l'écriture des textes (style, narration, structuration narrative), participation à une architecture d'ensemble, etc. Il est par ailleurs commun que le rassemblement conduise à l'échafaudage d'une narrativité englobante, les nouvelles se tissant pour produire la trame d'une seule histoire, aussi décousue ou parcellaire soit-elle ${ }^{23}$. C'est alors un processus accumulatif qui s'observe: les particules narratives proposées par chacune des nouvelles semblent s'arrimer, se compléter, étant de la sorte mobilisées dans une lecture globale de l'ouvrage. Certains de ces ouvrages sont parfois reçus comme des 
romans (ou des romans fragmentés), tant l'arrimage atténue les fractures entre les textes dans le cadre d'une lecture entière de l'œuvre.

Or, aussi étonnant que cela puisse paraître au premier abord, la dynamique narrative interne du recueil peut également être inverse. La multiplication des textes, des récits à l'intérieur d'un recueil n'est pas le gage d'un mouvement cohésif; cette pluralité peut engendrer tout autant un sentiment de désordre, d'éclatement, qui conduit à tout sauf à une lecture unifiante de l'ouvrage. On accolera généralement le qualificatif «hétérogènes» à ces recueils qui, à défaut de tendre vers une relative homogénéité, favorisent l'exposition brute des contrastes appelant une lecture différenciée des textes rassemblés ${ }^{24}$. Cette esthétique du recueil, loin de constituer une protestation contre la mise en récit des nouvelles, se construit en réalité par stratégie d'évitement: en ne misant pas sur les proximités narratives entre les textes, elle propose simplement un mode de relation autre entre eux.

Si une instrumentalisation de la narrativité peut s'observer au sein des pratiques polytextuelles, elle se bâtit contre ces attentes assez bien cadrées concernant la lecture du recueil de nouvelles ${ }^{25}$. Deux dérives par rapport à ces attentes peuvent être envisagées. La première se base sur l'a priori de la cohérence fictionnelle et narrative. S'il y a pour le lecteur des indices clairs d'un partage d'univers fictionnel entre les textes, il est attendu que les textes rassemblés se développent en une mosaïque coordonnée par un principe de cohérence: chacune des nouvelles contribue à la mise en place d'une perspective d'ensemble d'un univers et d'une trame narrative qui le mobilisera, perspective qui sera peut-être fragmentée, mais à tout le moins constituée d'éléments non contradictoires entre eux. Pourtant, en parcourant un ouvrage polytextuel comme Des anges mineurs d'Antoine Volodine (1999), tout lecteur sera pris par un doute sérieux: sa lecture de l'ensemble est-elle déficiente? Il en viendra tôt ou tard à postuler qu'il y a une incompatibilité entre les données narratives proposées par les quarante-neuf textes. Si la reconstruction de l'univers à partir de ses bribes constitue un défi en soi (peut-être in fine irréalisable), toute tentative de réconcilier les parcelles de récit qui jonchent l'ouvrage se butera à des incohérences manifestes: variations dans l'évaluation du temps passé depuis l'occurrence d'un événement, séquence dans laquelle surviennent des faits, chronologie sociohistorique indéterminable, multiplication incertaine des niveaux narratifs (comme la mise en scène hypothétique proposée par Maria Clementi [Volodine, 2001: 198-201])... De façon similaire à ce que l'on observe dans les hyperfictions, ces incompatibilités narratives franches ont pour effet de décentrer le récit de son rôle central au sein de cet ouvrage. Servant de support à l'établissement de "capsules fictionnelles", ces nouvelles (appelées intrafictionnellement des «narrats») sont rassemblées en un projet mobilisateur qui tient peut-être du roman, mais dont la trame narrative est inéluctablement déconstruite par l'impossibilité d'en saisir logiquement tous les fragments - contrairement à ce que nous laisse croire l'horizon d'attente du recueil. Si la fiabilité du narrateur principal est probablement en cause, celui-ci offrant une multiplicité de points de vue sur des faits qui se recoupent imparfaitement ${ }^{26}$, la conséquence demeure la même: la narrativité agit comme le canal de mise en place d'une fiction foisonnante, l'intégrité de l'œuvre n'étant pas liée au rétablissement hypothétique de sa cohérence.

La seconde dérive des attentes liées aux pratiques polytextuelles qui peut conduire à une instrumentalisation du narratif repose elle-même sur un déplacement, ici d'ordre discursif. La lecture d'un recueil de textes brefs, si elle rencontre événements, personnages et transformation des perceptions, conduira à postuler qu'il s'agit d'un recueil de nouvelles, avec le lot de présupposés et d'attentes qui lui sont liés. C'est à l'évidence ce qui peut guider le lecteur des recueils d'un Philippe Delerm, depuis La première gorgée de bière et autres plaisirs (1997) jusqu'à ses Enregistrements pirates (2004): en s'engageant dans ces ouvrages, il retrouve une voix narrative, souvent impersonnelle, mais qui le guide à travers des événements du quotidien, des situations singulières présentées dans leur caractère universel. L'inconfort s'insinue tranquillement: le narratif, constate-t-on, est 
contaminé par le tableau, par le commentaire, où la vérité générale s'exprime par le "on» (pour ne citer qu'un exemple, l'excipit du texte éponyme du premier recueil: "C'est un bonheur amer, on boit pour oublier la première gorgée» [1997: 32]). Désincarnée, cette narrativité glisse vers l'essai, auquel elle ne correspond pas de façon plus satisfaisante: présence d'une subjectivité du regard, mais effacement de l'identité du locuteur ${ }^{27}$; ton du commentaire sans accession à une réflexion réellement engagée. Par ce glissement des types discursifs, les ouvrages de Delerm ne parviennent pas à rejoindre les paramètres associés à nos conceptions tant de l'essai que de la prose narrative. Au-delà du questionnement de l'appartenance générique de ces textes, il apparaît clair que la narrativité qui les caractérise se présente comme l'instrument d'un projet qui n'est pas, de toute évidence, celui d'un récit - cette instrumentalisation, au demeurant, n'étant même pas dévouée à la cause de la fiction, qui est ici tout autant incertaine et fuyante. L'exemple de Delerm révise ainsi doublement le modèle canonique du roman: en plus de procéder à des déplacements génériques désarçonnants, ses textes ne voient pas la fiction et le récit se construire réciproquement et, encore moins, ils ne maintiennent le rôle structurant et signifiant de la narrativité puisqu'ils la réduisent à une fonction instrumentale: permettre à une voix de commenter des situations de la vie et de remettre en question des problèmes du quotidien.

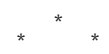

C'est à partir de l'examen de différentes pratiques littéraires que s'impose, avec le plus d'évidence, la nécessité de mieux cerner la notion de récit dans le discours critique. Terme (trop?) commun, employé pour désigner tant le type discursif que la configuration spécifique d'un texte en prose, le récit est appelé à être ressaisi pour être situé dans un usage plus opératoire. Il parait ainsi utile de définir plus précisément la narrativité, de l'associer à cette conception plus phénoménologique d'une événementialité, en dehors de sa réalisation principale, celle du récit (comme type de discours contraint par une grammaire idéalisée). Le mouvement narratif, plus large et moins spécifique que ce récit, peut ainsi être envisagé dans son interaction avec le paramètre qui lui est le plus communément associé, celui de la fictionnalité. Par leur étude dans une perspective communicationnelle, à la suite de Richard Walsh, il nous est ainsi possible d'en considérer les fonctions, les usages respectifs - et de prendre acte qu'ils n'interviennent pas dans des rôles constants ni dans un rapport équivalent. La narrativité, si elle est généralement structurante et porteuse du schème d'intelligibilité du texte (dans sa temporalité, dans sa téléologie), peut dans certaines circonstances être déclassée au profit d'un usage plus instrumental, qu'exemplifient les pratiques de l'essai, de l'hyperfiction et de certains ouvrages polytextuels. Cette traversée des rapports réciproques de la fiction et de la narrativité, de leur dialectique à géométrie variable, ouvre sur une compréhension moins monolithique des usages du discours narratif, et rejoint ainsi les témoignages de lecteurs d'œuvres contemporaines: en peinant à y reconnaître la présence de récit, ces lecteurs confirment la nécessité de le distinguer de la narrativité, catégorie discursive dont l'usage n'est pas unique ni fixe, et que des pratiques génériques peuvent très diversement investir. 


\section{N O TES}

1. D. Viart remet en question ce syntagme, signalant que ce n'est pas tant un retour $d u$ récit qu'un retour $a u$ récit - le récit n'a pas disparu, il s'est éclipsé. Il redevient un mode d'expression significatif après sa remise en question brutale par le Nouveau Roman et le textualisme. Viart note cependant avec justesse que le récit n'est plus aujourd'hui ce qu'il était, ce dont témoigne le collectif qu'il introduit (Mémoires $d u$ récit), où les contributions s'intéressent "aux formes prises par ce retour autant qu'aux modes de présence du récit dans l'écriture

contemporaine " (1998: 7).

2. On parle souvent de la pratique romanesque de Yergeau, mais on néglige souvent sa production quasi romanesque, si l'on peut dire, en référence à ses recueils de nouvelles à la frontière du roman, comme $T u$ attends la neige, Léonard? (1992) et Du virtuel à la romance (1999).

3. C'est à tout le moins l'impression que nous laissent les ouvrages cités, impression qui sera nuancée plus loin par les considérations anglo-saxonnes sur la fictionnalité.

4. Notre traduction: dorénavant NT. «W(S) is a closed, atemporal, Parmenidean realm of stillness and silence, where nothing changes, nothing happens" (Doležel, 1998: 32).

5. NT. «[...] formative operation, shapes narrative worlds into orders that have the potential to produce (generate) stories " (Doležel, 1998: 113). 6. NT. «[...] the class of discourse models used for understanding narratively organised discourse in particular » (Herman, John et Ryan, 2005: 569)

7. NT. «In trying to make sense of a narrative, interpreters attempt to reconstruct not just what happened but also the surrounding context or environment embedding storyworld existents, their attributes, and the actions and events in which they are involved " (Herman, John et Ryan, $2005:$ 570).

8. NT. «The Formalists perceived that fictional narrative is primarily driven by the dictates of art (however they might be conceived), and that its conformity to a logic of representation is always in some sense dissimulated. The implications of this specific interest in fictionality have been obscured in the subsequent history of narrative theory, however, precisely to the extent that the artifice involved [l'artifice qu'est la littérature] has come to be understood as an attribute of narrative in general» (Walsh, 2001: 594).

9. NT. «[...] narratives are constructs, and their meanings are internal to the system of narrative" (Walsh, 2005: 151).

10. NT. «Fictionality functions within a communicative framework: it resides in a way of using language, and its distinctiveness consists in the recognizably distinct rhetorical set invoked by that use " (Walsh, 2005: 152).

11. NT. «Fiction does not achieve relevance globally, at one remove, through some form of analogical thinking, but incrementally, through the implication of various cognitive interests or values that are not contingent upon accepting the propositional truth of the utterance itself; and upon the deployment, investment, and working through of those interests in narrative form " (Walsh, 2005: 158).

12. NT. «There is, certainly a global, retrospective sense in which narrative can be understood as the suspension of relevance along the line of action, and narrative closure figures less as the resolution of the plot in itself (though it is an effect usually achieved in terms of plot), than as the resolution of suspended evaluations of relevance. In this straightforward sense, irrespective of questions of fictionality, narrative form in itself responds to certain expectations of relevance» (Walsh, 2005: 158).

13. NT. «Narrativity refers preeminently to the way in which a narrative articulates itself, the way in which each stage of its own extension creates what might be called a crisis or dilemma of the discourse, which is solved by its own furtherance in whatever form that happens to take" (Sturgess, 1992: 26).

14. NT. «[...] the enabling force of narrative, a force that is present at every point in the narrative and thus always operates syntagmatically" (Sturgess, 1992: 28).

15. NT. « [...] to read a narrative with a sense of its wholeness means to read its narrativity. This reading will itself produce a story-the story of narrativity-which will enhance, dramatize and above all provide a rationale for the story being told" (Sturgess, 1992: 28).

16. NT. "The set of properties characterizing narrative and distinguishing it from nonnarrative; the formal and contextual features making a (narrative) text more or less narrative, as it were " (Prince, 2003: 65).

17. NT. «What narrativity seems primarily linked to is general narrative configuration (as opposed to specific thematic traits, contextual pointedness, or textual value). More particularly, it seems linked to the formal nature of the narrated (e.g. structural closure), that of the narrating (e.g. foregrounding of event discreteness) and the relations between the two (e.g. no inordinate amount of commentary)» (Prince, 1999: 48).

18. Voir à ce propos les réflexions d'ordre philosophique proposées par C. Romano (1999).

19. En ce sens, cet usage de la notion d'événement se distingue singulièrement de l'usage plus commun en théorie du récit, que résument J.-M. Adam et $\mathrm{F}$. Revaz: «le récit est l'exposé des "faits" réels ou imaginaires. La désignation générale de "faits" recouvre deux réalités qu'il est utile de distinguer: l'ÉVÉNEMENT et l'ACTION. [...] l'ACTION se caractérise par la présence d'un AGENT [...] qui provoque le changement (ou tente de l'empêcher), tandis que l'ÉVÉNEMENT advient sous l'effet de CAUSES, sans intervention intentionnelle d'un agent" (1996: 14).

20. Notons ici qu'il sera fait allusion à des postures de lecture conventionnelles, en ce sens qu'elles correspondent aux pratiques lecturales telles qu'elles sont associées à des genres ou à des discours, ceux-ci appelant un spectre spécifique d'attentes. S'il y a convention, il y a par ailleurs des exceptions, des pratiques marginales - toutes les lectures ne sont évidemment pas représentées par ces efforts de généralisation de la réception des œuvres, mais elles correspondront à un degré plus ou moins éloigné à ces postures de lecture régies par des conventions génériques.

21. Sur la question de l'intégration de la fiction dans l'essai, on peut notamment se référer à «La fiction à l'essai" (Audet, 2002).

22. Cette désorientation des lecteurs, bien documentée dans les études sur la littérature électronique, a notamment fait l'objet d'analyses empiriques par David S. Miall (dont il a fait le bilan en lien avec la théorisation de l'hypertexte [1999]); une démonstration par la négative de cette perplexité des lecteurs repose sur la difficulté manifeste qu'ont les œuvres électroniques à percer auprès d'un public large et diversifié (même si d'autres facteurs, d'accessibilité technique et de diffusion, sont certes à prendre en considération).

23. Tant l'essai que la poésie et la nouvelle, dans leur assemblage en un livre, peuvent élaborer une narrativité d'ensemble; pour un aperçu des modalités possibles de narrativisation pour chacune de ces pratiques, voir R. Audet et T. Bissonnette (2004).

24. A. Carpentier a défendu à quelques reprises l'idée (et l'intérêt) du recueil hétérogène: «Ce contre quoi je m'élève, c'est le vice éditorial qui consiste à commander des recueils tendant vers la complétude, des 
recueils structurés comme des romans. Le cas limite étant le recueil carrément déguisé en roman, portant le masque de cette inscription générique. [...] contre la plupart des critiques en attente de systèmes à l'emporte-pièce, par lesquels ils reconnaissent les œuvres bien nées, je dois admettre que j'aime assez ces recueils délinquants qui refusent de feindre un ordre, qui renoncent au mensonge du système [...], ces recueils qui exigent, tout autant que les recueils homogènes, des lecteurs songeant à construire, mais un peu plus par eux-mêmes " (1993: 45).

25. Pour un examen des processus de lecture impliqués par la pratique du recueil de nouvelles, on pourra se reporter à Audet (2000).

26. On pourrait évoquer ici le cas du roman 1999 de P. Yergeau (1995), qui met en place une dynamique similaire: pluralité de points de vue divergents sur une même série de faits, histoires multipliées mais pas nécessairement compatibles... Voir à ce sujet A.-M. Clément, 2002 : 99. 130 .

27. Dans le même paradigme que Delerm mais avec des voix nettement plus affirmées, signalons le Palomar d'I. Calvino (1983), à mi-chemin entre le recueil, le roman et l'essai, et À l'heure du loup de P. Morency (2002), qui ajoute une touche poétique au mélange déjà insaisissable de fiction, d'essai et de narrativité fuyante.

\section{RÉFÉREN CES BIBLIO G RAPHIQ U ES}

ADAM, J.-M. [1994]: "Décrire des actions: raconter ou relater?", Littérature, n०95, 3-22;

— [1999]: Le Récit, Paris, PUF, coll. "Que sais-je?».

ADAM, J.-M. et F. REVAZ [1996] : L’Analyse des récits, Paris, Seuil. AUDET, R. [2000]: Des textes à l'œuvre. La lecture du recueil de nouvelles, Québec, Nota bene;

[2002]: "La fiction à l'essai», dans A. Gefen et R. Audet (dir.), Frontières de la fiction, Québec et Bordeaux, Nota bene et Les Presses universitaires de Bordeaux, 133-157;

— [2006] : "La narrativité est affaire d'événement", dans D. Rivière (dir.), Jeux et enjeux de la narrativité dans les pratiques contemporaines, Paris, Éd. Dis voir, 7-35.

AudeT, R. et T. BissonneTte [2004]: «Le recueil littéraire, une variante formelle de la péripétie», dans R. Audet et A. Mercier (dir.), La Narrativité contemporaine au Québec. 1. La littérature et ses enjeux narratifs, Québec, Presses de l'Université Laval, 15-43.

BARTHES, R. (dir.) [1966]: Dossier «Recherches sémiologiques: analyse structurale du récit", Communications, $\mathrm{n}^{\circ} 8$.

BraulT, J. [1991]: Ô saisons, ô châteaux, Montréal, Boréal, coll. «Papiers collés".

Calvino, I. [(1983) 1985]: Palomar, Paris, Seuil.

CARPENTIER, A. [1993]: "Commencer et finir souvent. Rupture fragmentaire et brièveté discontinue dans l'écriture nouvellière", dans A. Whitfield et J. Cotnam (dir.), La Nouvelle: écriture(s) et lecture(s), Toronto et Montréal, Éd. du GREF et XYZ, 35-48.

Chevillard, É. [2003]: Le Vaillant Petit Tailleur, Paris, Minuit. ClÉment, A.-M. [2005] : «Formes et sens de la discontinuité dans la prose narrative québécoise contemporaine", thèse de doctorat, Université Laval.

COHN, D. [1999]: The Distinction of Fiction, Baltimore, The Johns Hopkins University Press.

DELERM, P. [1997] : La première gorgée de bière et autres plaisirs minuscules, Paris, Gallimard;

— [2004] : Enregistrements pirates, Paris, Éd. du Rocher.
DoležEl, L. [1998]: Heterocosmica. Fiction and Possible Worlds, Baltimore, The Johns Hopkins University Press.

GeneTte, G. [1991] : "Récit fictionnel, récit factuel ", Fiction et Diction, Paris, Seuil, 65-94.

Greimas, A. J. et J. COURTÉs [1979]: Sémiotique. Dictionnaire raisonné de la théorie du langage, tome 1, Paris, Hachette.

GRICE, H. P. [1975]: "Logic and Conversation ", dans D. Davidson et G. Harman (dir.), The Logic of Grammar, Encino, Dickenson, 64-75. Hamburger, K. [(1957) 1986]: Logique des genres littéraires, Paris, Seuil. Herman, D. [2002]: Story Logic. Problems and Possibilities of Narrative, Lincoln, University of Nebraska Press.

Herman, D., M. JAhn et M.-L. RYAN (dir.) [2005]: The Routledge Encyclopedia of Narrative Theory, New York, Routledge.

LARIVAille, P. [1974]: "L'analyse (morpho)logique du récit», Poétique, $\mathrm{n}^{\circ} 19,368-388$.

MiALL, D. S. [1999]: «Trivializing or Liberating? The Limitations of Hypertext Theorizing", Mosaic, vol.32, no2 (juin), 157-171. MOREnCY, P. [2002] : À l'heure du loup, Montréal, Boréal.

PRATT, M.-L. [1977]: Toward a Speech Act Theory of Literary Discourse, Bloomington, Indiana University Press.

PrincE, G. [1973] : A Grammar of Stories, La Haye, Mouton; [(1987) 2003]: Dictionary of Narratology, Lincoln, University of Nebraska Press; — [1999]: "Revisiting Narrativity», dans W. Grünweig et A. Solbach (dir.), Grenzüberschreitungen: Narratologie im Kontext / Transcending Boundaries: Narratology in Context, Tübingen, Gunter Narr Verlag, 43-51.

RABATÉ, D. [1991]: Vers une littérature de l'épuisement, Paris, José Corti. Ricceur, P. [1983]: Temps et Récit, Paris, Seuil.

Rimmon-Kenan, S. [1983]: Narrative Fiction, Londres, Methuen. Romano, C. [1999]: L'Événement et le Monde, Paris, PUF. RYAN, M.-L. [1991]: Possible Worlds, Artificial Intelligence, and Narrative Theory, Bloomington, Indiana University Press. SARraute, N. [1939]: Tropismes, Paris, Denoël [1997]: Ouvrez, Paris, Gallimard.

ScHOLES, R. [1982]: Semiotics and Interpretation, New Haven, Yale University Press.

SEARLE, J. [1969]: Speech Acts. An Essay in the Philosophy of Language, New York, Cambridge University Press.

Soucy, G. [(1997) 2001] : L'Acquittement, Montréal, Boréal. SPERBER, D. et D. WILSON [1984]: Relevance: Communication and Cognition, Oxford/Cambridge, Blackwell/Harvard University Press. Sturgess, P. J. M. [1992] : Narrativity: Theory and Practice, Oxford, Oxford University Press.

VIART, D. [1998] : "Mémoires du récit: questions à la modernité", dans D. Viart (dir.), Mémoires du récit, Paris, Minard / Lettres modernes, 3-27. Volodine, A. [1999]: Des anges mineurs, Paris, Seuil.

WaLsh, R. [2001]: "Fabula and Fictionality in Narrative Theory", Style, vol. 35, no4 (hiver), 592-606;

[2005]: "The Pragmatics of Narrative Fictionality ", dans

J. Phelan et P. J. Rabinowitz (dir.), A Companion to Narrative Theory, Oxford, Blackwell, 150-164.

White, H. [1975]: Metahistory. The Historical Imagination in NineteenthCentury Europe, Baltimore, The Johns Hopkins University Press. YergeaU, P. [1992]: Tu attends la neige, Léonard?, Québec, L'Instant même;

[1995]: 1999, Québec, L'Instant même;

— [1999]: Du virtuel à la romance, Québec, L'Instant même. 